\title{
Almost-sure path properties of fractional Brownian sheet
}

\author{
Wensheng Wang ${ }^{1}$ \\ Department of Statistics, East China Normal University, Shanghai 200062, China \\ and \\ Department of Mathematics, Hangzhou Teachers College, Hangzhou 310036, China
}

Received 6 October 2005; received in revised form 11 July 2006; accepted 12 September 2006

Available online 12 January 2007

\begin{abstract}
The almost sure sample function behavior of the vector-valued fractional Brownian sheet is investigated. In particular, the global and the local moduli of continuity of the sample functions are studied. These results give precise information about the continuity and the oscillation behavior of the sample functions.
\end{abstract}

(c) 2007 Elsevier Masson SAS. All rights reserved.

\section{Résumé}

On étudie le comportement p.s. du mouvement brownien fractionnaire vectoriel, en particulier ses modules de continuité local et global.

๑ 2007 Elsevier Masson SAS. All rights reserved.

Keywords: Gaussian process; Fractional Brownian sheet; Modulus of continuity; Law of the iterated logarithm

\section{Introduction}

The parameter space is $\mathbb{R}_{+}^{N}=[0, \infty)^{N}$; a typical parameter ("time points") is $t=\left(t_{1}, \ldots, t_{N}\right)$, sometimes also written as $\left\langle t_{i}\right\rangle$, or $\langle C\rangle$, if $t_{1}=\cdots=t_{N}=C$. For $s=\left\langle s_{i}\right\rangle, t=\left\langle t_{i}\right\rangle$ two points of $\mathbb{R}_{+}^{N}$ with $s_{i} \leqslant t_{i}, 1 \leqslant i \leqslant N,[s, t]$ denotes a $N$-dimensional interval of the type

$$
[s, t]=\times_{i=1}^{N}\left[s_{i}, t_{i}\right] .
$$

The $N$-dimensional Lebesgue measure is denoted by $\lambda$. Following Orey and Pruitt [9], we denote the Lebesgue measure $\lambda([\langle 0\rangle, s] \Delta[\langle 0\rangle, t])$ of the symmetric difference of two intervals $[\langle 0\rangle, s],[\langle 0\rangle, t]$ by $\delta(s, t)$, and simply by $\delta(t)$ in case $s=\langle 0\rangle: \delta(t)=\lambda([\langle 0\rangle, t])$.

The state space $\mathbb{R}^{d}$, is endowed with the Euclidean norm $\|\cdot\|$. Given a function $f: \mathbb{R}_{+}^{N} \rightarrow \mathbb{R}^{d}$, the "increment $f(Q)$ of $f$ over the interval $Q$ " is defined in just the same way as if $f$ were a distribution function and $f(Q)=\int_{Q} \mathrm{~d} f$ the $f$-measure of $Q$, e.g.

$$
f\left(\left[\left\langle s_{i}\right\rangle,\left\langle t_{i}\right\rangle\right]\right)=f\left(t_{1}, t_{2}\right)-f\left(t_{1}, s_{2}\right)-f\left(s_{1}, t_{2}\right)+f\left(s_{1}, s_{2}\right)
$$

\footnotetext{
E-mail address: wswang@ @stat.ecnu.edu.cn.
}

1 The author would like to acknowledge the partial support from the Natural Science Foundation of China, grant No. 10401037. 
in case $N=2$.

Now, to introduce the processes to be studied, let $X^{(N)}:=\left\{X_{t}^{(N)}, t \in \mathbb{R}_{+}^{N}\right\}, N \geqslant 1$, be a $\alpha$-fractional Brownian sheet taking values in $\mathbb{R}^{1}$, where $\alpha=\left(\alpha_{1}, \ldots, \alpha_{N}\right) \in(0,1)^{N}$, i.e. $X^{(N)}$ is a mean zero real-valued Gaussian process indexed by $N$-dimensional time with $X_{t}=0$ with probability one for all $t$ lying on the boundary of $\mathbb{R}_{+}^{N}$, that is with $\delta(t)=0$; whose covariance function is:

$$
E\left[X_{s}^{(N)} X_{t}^{(N)}\right]=c_{\alpha, N} \prod_{i=1}^{N}\left(\left|s_{i}\right|^{2 \alpha_{i}}+\left|t_{i}\right|^{2 \alpha_{i}}-\left|s_{i}-t_{i}\right|^{2 \alpha_{i}}\right),
$$

where $c_{\alpha, N}$ is a positive constant depending only on $\alpha$ and $N$. If $\alpha_{1}=\cdots=\alpha_{N}=1 / 2, X^{(N)}$ is usually referred as Brownian sheet or $N$-parameter Wiener process; we denote it by $W^{(N)}$ or simply $W . \alpha$-fractional Brownian sheet is a natural generalization of $N$-parameter Wiener process.

We denote $\alpha$-fractional Brownian sheet taking values in $\mathbb{R}^{d}, d \geqslant 1$, by $X^{(N, d)}$ or simply $X$. In case $N=1, d=1$, $X$ is the standard fractional Brownian motion. Throughout this paper, without loss of generality, we assume the constant in (1.1) $c_{\alpha, N}=2^{-N}$ so that $E\left[\left(X_{t}^{(N)}\right)^{2}\right]=\prod_{i=1}^{N} t_{i}^{2 \alpha_{i}}$.

The $N$-parameter Wiener process $W^{(N)}$ have been investigated by several authors. For more information about $W^{(N)}$ we refer to Park [11], Pyke [12], Orey and Pruitt [9] and Ehm [5].

Recently, some sample-function behavior of the $\alpha$-fractional Brownian sheet have been studied by several authors as natural generalization of Brownian sheet. Dunker [4], Mason and Shi [7] and Belinsky and Linde [1] investigated small ball probabilities for $X^{(N, 1)}$. Lu, Wang and Zhang [6] investigated large increment properties for $X^{(N, 1)}$ in case $N=2, \alpha_{1}=\alpha_{2}$.

In this paper we study the almost-sure sample path properties of the vector-valued fractional Brownian sheet with index $\alpha=\left(\alpha_{1}, \ldots, \alpha_{N}\right)\left(0<\alpha_{i}<1,1 \leqslant i \leqslant N\right)$. We show the global and the local continuity moduli of the sample functions. These results give precise information about the continuity and the small fluctuations of the sample functions.

As convention, to prove the continuity moduli, we can follow the classic method (cf. Theorem 1.1.1 in Csörgó and Révész [3]) without too much difficulty. The main points that require some care is the choice of the manner of discretizing the unit time interval for various problems and solve some dependence-related problems. In order to solve dependence problems and to prove its existence we give a concrete representation of $\alpha$-fractional Brownian sheet (see Section 2). Such a representation is based on the idea of Talagrand's (stochastic) integral representation for fractional Brownian motion (cf. Talagrand [13]. In fact, Talagrand [13] gave a representation for the fractional Lévy's $N$-parameter Brownian motion, a different type of processes (Gaussian vector fields).)

The paper is organized as follows. In Section 2 we give several preliminary properties and results for the $\alpha$-fractional Brownian sheet, which will be used in latter sections. The global and the local continuity moduli for $X^{(N, d)}$ are discussed in Sections 3 and 4 respectively.

Throughout this paper, finite and positive constants whose values are unimportant will be denoted by $c$. For $s, t \in \mathbb{R}_{+}^{N}, s \leqslant t$ (resp. $s<t$ ) means that $s_{i} \leqslant t_{i}$ (resp. $s_{i}<t_{i}$ ) for all $1 \leqslant i \leqslant N$. For $x \in \mathbb{R}, \operatorname{let} \log x=\ln (x)$, $\log \log x=\ln (\ln (x))$.

\section{Preliminaries}

In this section we shall give several preliminary results for $X$ that we need. We first state several useful facts for $X^{(N)}$ (for intervals) the $\alpha$-fractional Brownian sheet taking values in $\mathbb{R}^{1}$. The first one is on the covariance function for intervals.

(i) For any intervals $[s, t],\left[s^{\prime}, t^{\prime}\right] \subset \mathbb{R}_{+}^{N}$

$$
E\left[X^{(N)}([s, t]) X^{(N)}\left(\left[s^{\prime}, t^{\prime}\right]\right)\right]=\frac{1}{2^{N}} \prod_{i=1}^{N}\left(-\left|t_{i}-t_{i}^{\prime}\right|^{2 \alpha_{i}}+\left|t_{i}-s_{i}^{\prime}\right|^{2 \alpha_{i}}+\left|s_{i}-t_{i}^{\prime}\right|^{2 \alpha_{i}}-\left|s_{i}-s_{i}^{\prime}\right|^{2 \alpha_{i}}\right) .
$$

(ii) Self-similarity: The processes $\left(\prod_{i=1}^{N} C_{i}^{-\alpha_{i}}\right) X_{\left\langle C_{i} t_{i}\right\rangle}^{(N)}$ and $X_{\left\langle t_{i}\right\rangle}^{(N)},\left\langle t_{i}\right\rangle \in \mathbb{R}_{+}^{N}$, are identical in distribution, for every $\left\langle C_{i}\right\rangle \in(0, \infty)^{N} ; X^{(N)}([s, t])$ is distributed like $\left(\prod_{i=1}^{N}\left(t_{i}-s_{i}\right)^{\alpha_{i}}\right) X_{\langle 1\rangle}^{(N)}$ for every interval $[s, t]$. 
(iii) Stationary increments: The processes $X^{(N)}\left(\left[\left\langle C_{i}\right\rangle+s,\left\langle C_{i}\right\rangle+t\right]\right)$ and $X^{(N)}([s, t]), s, t \in \mathbb{R}_{+}^{N}$, are identical in distribution, for every $\left\langle C_{i}\right\rangle \in[0, \infty)^{N}$.

(iv) Additivity: For any real number $s, s_{0}, s^{\prime}$ such that $s \leqslant s_{0} \leqslant s^{\prime}$ and any $t, t^{\prime} \in \mathbb{R}_{+}^{N-1}, X^{(N)}\left(\left[(s, t),\left(s^{\prime}, t^{\prime}\right)\right]\right) \equiv$ $X^{(N)}\left(\left[(s, t),\left(s_{0}, t^{\prime}\right)\right]\right)+X^{(N)}\left(\left[\left(s_{0}, t\right),\left(s^{\prime}, t^{\prime}\right)\right]\right)$. In case $s=s^{\prime}, X\left(\left[(s, t),\left(s^{\prime}, t^{\prime}\right)\right]\right) \equiv 0$.

(v) The processes $X^{(N)}\left(\left[(0, t),\left(1, t^{\prime}\right)\right]\right)$ and $X^{(N-1)}\left(\left[t, t^{\prime}\right]\right), t, t^{\prime} \in \mathbb{R}_{+}^{N-1}$, are identical in distribution, where the latter process is the $\left(\alpha_{2}, \ldots, \alpha_{N}\right)$-fractional Brownian sheet.

Remark 2.1. For increments of the form $X_{t}^{(N)}-X_{s}^{(N)}$ (iii) is completely false. In particular if $s=\left\langle s_{i}\right\rangle, t=\left\langle s_{i}+u_{i}\right\rangle$, the variance $X_{t}^{(N)}-X_{s}^{(N)}$ will depend crucially on $s$.

Now we establish some Fernique type inequalities for $X^{(N, d)}$. Let now, as well as in the rest paper, for brevity's sake, $X:=X^{(N, d)}$.

Lemma 2.1. For any $0<\varepsilon<1$ there exists $c=c(\varepsilon, \alpha, N, d)$ such that

$$
P\left\{\sup _{\left\langle x_{i}\right\rangle \leqslant t \leqslant\left\langle x_{i}+T_{i}\right\rangle\langle 0\rangle \leqslant s \leqslant\left\langle a_{i}\right\rangle} \sup _{\langle x}\|X([t, t+s])\| \geqslant u \prod_{i=1}^{N} a_{i}^{\alpha_{i}}\right\} \leqslant c\left(\prod_{i=1}^{N}\left(\frac{T_{i}}{a_{i}}+1\right)\right) \mathrm{e}^{-u^{2} /(2+\varepsilon)}
$$

for every $u \geqslant u_{0}>0,\left\langle x_{i}\right\rangle \in \mathbb{R}_{+}^{N}$, and $\left\langle T_{i}\right\rangle,\left\langle a_{i}\right\rangle \in \mathbb{R}_{+}^{N}$. Particularly, for any $0<\varepsilon<1$ there exists $c=c(\varepsilon, \alpha, N, d)$ such that

$$
P\left\{\sup _{R \subset\left[\left\langle x_{i}\right\rangle,\left\langle x_{i}+a_{i}\right\rangle\right]}\|X(R)\| \geqslant u \prod_{i=1}^{N} a_{i}^{\alpha_{i}}\right\} \leqslant c \mathrm{e}^{-u^{2} /(2+\varepsilon)}
$$

for every $u \geqslant u_{0}>0,\left\langle x_{i}\right\rangle,\left\langle a_{i}\right\rangle \in \mathbb{R}_{+}^{N}$, where $R=\left[\left\langle y_{i}\right\rangle,\left\langle z_{i}\right\rangle\right]$ ranges over intervals.

Proof. We first show (2.2). By stationary increments for intervals (see (iii)), we can assume that $\left\langle x_{i}\right\rangle=\langle 0\rangle$. For notation simplicity only, we give the proof for $N=1$ and $=2$. The following fact will be used in the proof: Let $U$ be a normal random variable in $\mathbb{R}^{d}$ with mean zero and identity covariance matrix. Then

$$
c_{1}(d) u^{d-2} \mathrm{e}^{-u^{2} / 2} \leqslant P(\|U\| \geqslant u) \leqslant c_{2}(d) u^{d-2} \mathrm{e}^{-u^{2} / 2}
$$

for any $u \geqslant u_{0}>0$. (Since $\|U\|^{2}$ has a $\chi^{2}$ distribution with $d$ degrees of freedom.)

In case $N=1$, by (2.4), (2.2) is an immediate consequence of Lemma 2.4 of Csáki, Csörgő and Shao [2]. For case $N=2$, by using (iv) and (2.4), the proof is similar to that of Lemma 2.5 of Lu, Wang and Zhang [6]. Here we give a sketch of the proof for the sake of completeness. For any positive real number $t$ and $r$ put $t_{r}=a_{1}\left[t \cdot\left(2^{2^{r}} / a_{1}\right)\right] / 2^{2^{r}}$. By (iv) we have

$$
\begin{aligned}
\|X([x, x+s] \times[y, y+t])\| \leqslant & \left\|X\left(\left[x_{r},(x+s)_{r}\right] \times[y, y+t]\right)\right\|+\left\|X\left(\left[(x+s)_{r}, x+s\right] \times[y, y+t]\right)\right\| \\
& +\left\|X\left(\left[x_{r}, x\right] \times[y, y+t]\right)\right\| \\
\leqslant & \left\|X\left(\left[x_{r},(x+s)_{r}\right] \times[y, y+t]\right)\right\| \\
& +\sum_{j=0}^{\infty}\left\|X\left(\left[(x+s)_{r+j},(x+s)_{r+j+1}\right] \times[y, y+t]\right)\right\| \\
& +\sum_{j=0}^{\infty}\left\|X\left(\left[x_{r+j}, x_{r+j+1}\right] \times[y, y+t]\right)\right\| .
\end{aligned}
$$

Using (2.4) and repeating the lines of the proof of Lemma 2.1 of Csáki, Csörgó and Shao [2], we have for any $y, t \in \mathbb{R}_{+}^{1}$,

$$
P\left\{\sup _{0 \leqslant x \leqslant T_{1}} \sup _{0 \leqslant s \leqslant a_{1}}\|X([x, x+s] \times[y, y+t])\| \geqslant u a_{1}^{\alpha_{1}} t^{\alpha_{2}}\right\} \leqslant c\left(\frac{T_{1}}{a_{1}}+1\right) \mathrm{e}^{-u^{2} /(2+\varepsilon)} .
$$


By using (2.5) and proceeding to the same lines of the above proof for the second coordinate, we have

$$
P\left\{\sup _{\substack{0 \leqslant x \leqslant T_{1} \\ 0 \leqslant y \leqslant T_{2}}} \sup _{\substack{0 \leqslant s \leqslant a_{1} \\ 0 \leqslant t \leqslant a_{2}}}\|X([x, x+s] \times[y, y+t])\| \geqslant u a_{1}^{\alpha_{1}} a_{2}^{\alpha_{2}}\right\} \leqslant c\left(\frac{T_{1}}{a_{1}}+1\right)\left(\frac{T_{2}}{a_{2}}+1\right) \mathrm{e}^{-u^{2} /(2+\varepsilon)} .
$$

This proves (2.2).

By choosing $T_{i}=a_{i}$ for all $i$ in (2.2), we get (2.3) immediately. The proof of Lemma 2.1 is complete.

We conclude this section by giving a concrete representation of the process $X^{(N)}$, which is very useful to show the existence of $X^{(N)}$ and to solve dependence problems involved in Section 4.

Let $W^{(N)}(x), x \in \mathbb{R}^{N}$, be a $N$-parameter Wiener process. Then, we assert that the process

$$
X_{t}^{(N)}=\int_{\mathbb{R}^{N}} \prod_{i=1}^{N} \frac{c\left(\alpha_{i}\right)\left(1-\cos \left(t_{i} x_{i}\right)+\sin \left(t_{i} x_{i}\right)\right)}{\left|x_{i}\right|^{\alpha_{i}+1 / 2}} \mathrm{~d} W^{(N)}(x)
$$

is a version of $\alpha$-fractional Brownian sheet, where

$$
c\left(\alpha_{i}\right)=\left(2 \int_{\mathbb{R}}(1-\cos x) \frac{\mathrm{d} x}{|x|^{2 \alpha_{i}+1}}\right)^{-1 / 2} .
$$

(It is a positive and finite constant depending only on $\alpha_{i}$.) The definition of stochastic integral with respect to $N$-parameter Wiener process was given by Park [11].

In fact, such a representation is based upon the fact that if $0<H<1$, there is a constant $c(H)$ depending upon $H$ only such that for each $t$ in $\mathbb{R}$, by change of variable,

$$
|t|^{2 H}=c(H)^{2} \int_{\mathbb{R}} 2(1-\cos (t x)) \frac{\mathrm{d} x}{|x|^{2 H+1}} .
$$

To simplify notation, let

$$
f(H, t, x)=\frac{c(H)(1-\cos (t x)+\sin (t x))}{|x|^{H+1 / 2}} .
$$

Then

$$
f(H, t, x)^{2}=c(H)^{2}[2(1-\cos (t x))+2(1-\cos (t x)) \sin (t x)] /|x|^{2 H+1},
$$

and so $\int_{\mathbb{R}} f(H, t, x)^{2} \mathrm{~d} x=|t|^{2 H}$. Thus, using the relation

$$
f(H, t, x) f(H, s, x)=\frac{1}{2}\left[f(H, t, x)^{2}+f(H, s, x)^{2}-(f(H, t, x)-f(H, s, x))^{2}\right],
$$

it holds

$$
\int_{\mathbb{R}} f(H, t, x) f(H, s, x) \mathrm{d} x=\frac{1}{2}\left(|t|^{2 H}+|s|^{2 H}-|t-s|^{2 H}\right) .
$$

By Lemma 3 of Park [11] (see (4.15) on page 1588) it holds

$$
\begin{aligned}
E\left[X_{t}^{(N)} X_{s}^{(N)}\right] & =\int_{\mathbb{R}^{N}} \prod_{i=1}^{N} f\left(\alpha_{i}, t_{i}, x_{i}\right) f\left(\alpha_{i}, s_{i}, x_{i}\right) \mathrm{d} x_{1} \cdots \mathrm{d} x_{N}=\prod_{i=1}^{N} \int_{\mathbb{R}} f\left(\alpha_{i}, t_{i}, x_{i}\right) f\left(\alpha_{i}, s_{i}, x_{i}\right) \mathrm{d} x_{i} \\
& =2^{-N} \prod_{i=1}^{N}\left(\left|t_{i}\right|^{2 H}+\left|s_{i}\right|^{2 H}-\left|t_{i}-s_{i}\right|^{2 H}\right) .
\end{aligned}
$$

This verifies (1.1) and concludes the assertion. 


\section{Global moduli of continuity}

In this section we shall show the global continuity moduli for the $\alpha$-fractional Brownian sheet $X:=X^{(N, d)}$.

Theorem 3.1 (Global continuity modulus for intervals). Let

$$
\begin{aligned}
& d(s, t)=\prod_{i=1}^{N}\left(t_{i}-s_{i}\right)^{\alpha_{i}}, \quad \lambda([s, t])=\prod_{i=1}^{N}\left(t_{i}-s_{i}\right), \\
& \beta(s, t)=d(s, t)|\log \lambda([s, t])|^{1 / 2}, \quad s, t \in \mathbb{R}_{+}^{N} .
\end{aligned}
$$

Then

$$
\lim _{\varepsilon \rightarrow 0} \sup _{s, t \in[0,1]^{N}, \lambda([s, t]) \leqslant \varepsilon} \beta(s, t)^{-1}\|X([s, t])\|=2^{1 / 2} \quad \text { a.s. }
$$

Remark 3.1. For a standard (one-dimensional) Wiener process, the form of the continuity modulus in Theorem 3.1 is slightly stronger than the classical Lévy's form because, the normalized factor is $\sqrt{|t-s||\log | t-s||}$, while in the latter form that is $\sqrt{\varepsilon|\log \varepsilon|}$.

Proof. At first, we show

$$
\limsup _{\varepsilon \rightarrow 0} I(\varepsilon) \leqslant 2^{1 / 2} \quad \text { a.s., }
$$

where

$$
I(\varepsilon)=\sup _{s, t \in[0,1]^{N}, \lambda([s, t]) \leqslant \varepsilon} \beta(s, t)^{-1}\|X([s, t])\| .
$$

Let $i=\left(i_{1}, \ldots, i_{N-1}\right), m=\left(m_{1}, \ldots, m_{N-1}\right)$, and define the events for $\mu>0$

$$
E(i, j, k, m, n)=\left[\sup \|X([s, t])\| \geqslant(1+\mu) A \sqrt{2 \log \left(B^{-1}\right)}\right],
$$

where the supremum is taken over all $s, t$ satisfying

$$
\begin{aligned}
& \frac{i_{p}}{n} 2^{-m_{p} / n} \leqslant s_{p}<\frac{i_{p}+1}{n} 2^{-m_{p} / n}, \quad p=1, \ldots, N-1, \\
& \frac{i_{p}}{n} 2^{-m_{p} / n}+2^{-\left(m_{p}+1\right) / n} \leqslant t_{p}<\frac{i_{p}+1}{n} 2^{-m_{p} / n}+2^{-m_{p} / n}, \quad p=1, \ldots, N-1 ; \\
& \frac{j-1}{2^{n}} \leqslant s_{N}<\frac{j}{2^{n}}, \quad \frac{j+k}{2^{n}} \leqslant t_{N}<\frac{j+k+1}{2^{n}},
\end{aligned}
$$

and

$$
A=A(\alpha, k, m, n)=\left(2^{-1 / n}-n^{-1}\right)^{\alpha_{1}+\cdots+\alpha_{N-1}} 2^{-\left(\alpha_{1} m_{1}+\cdots+\alpha_{N-1} m_{N-1}\right) / n}\left(k 2^{-n}\right)^{\alpha_{N}}
$$

is the infimum of $d(s, t)$ for the intervals involved, and

$$
A^{\prime}=A^{\prime}(\alpha, k, m, n)=\left(1+n^{-1}\right)^{\alpha_{1}+\cdots+\alpha_{N-1}} 2^{-\left(\alpha_{1} m_{1}+\cdots+\alpha_{N-1} m_{N-1}\right) / n}\left((k+2) 2^{-n}\right)^{\alpha_{N}}
$$

is the corresponding supremum, and

$$
B=B(\alpha, k, m, n)=\left(1+n^{-1}\right)^{N-1} 2^{-\left(m_{1}+\cdots+m_{N-1}\right) / n}(k+2) 2^{-n}
$$

is the supremum of $\lambda([s, t])$ for the intervals involved. The parameters will be restricted to the following ranges:

$$
0 \leqslant i_{p} \leqslant n 2^{m_{p} / n}, \quad 1 \leqslant j \leqslant 2^{n}, \quad \frac{1}{4} n \leqslant k \leqslant n, \quad 0 \leqslant m_{p}<n^{2},
$$

$p=1, \ldots, N-1, n=3,4, \ldots$ It is easy to check that

$$
0 \leqslant 1-A\left(A^{\prime}\right)^{-1} \leqslant c n^{-1} \rightarrow 0 .
$$


By (2.3) (with $\left.u=(1+\mu) A\left(A^{\prime}\right)^{-1} \sqrt{2 \log \left(B^{-1}\right)}\right)$ we obtain

$$
\begin{aligned}
P(E(i, j, k, m, n)) & \leqslant c \exp \left[-\left(1+\frac{\mu}{2}\right)^{2} \log \left(B^{-1}\right)\right] \\
& \leqslant c 2^{-(1+\mu / 4)^{2}\left(m_{1}+\cdots+m_{N-1}\right) / n} 2^{-(1+\mu / 4)^{2} n}
\end{aligned}
$$

for large $n$. Thus

$$
\begin{aligned}
\sum_{n} \sum_{i, j, k, m} P(E(i, j, k, m, n)) & \leqslant c \sum_{n} \sum_{i, j, k, m} 2^{-(1+\mu / 4)^{2}\left(m_{1}+\cdots+m_{N-1}\right) / n} 2^{-(1+\mu / 4)^{2} n} \\
& \leqslant c \sum_{n} \sum_{m} n 2^{-\left((1+\mu / 4)^{2}-1\right)\left(m_{1}+\cdots+m_{N-1}\right) / n} 2^{-\left((1+\mu / 4)^{2}-1\right) n} \\
& \leqslant c \sum_{n=1}^{\infty} n^{2 N-1} 2^{-\left((1+\mu / 4)^{2}-1\right) n}<\infty
\end{aligned}
$$

where $\sum_{i, j, k, m}, \sum_{m}$ take over the above ranges of $i, j, k, m$. Thus, by Borel-Cantelli lemma, a.s. only finitely many of the events $E(i, j, k, m, n)$ occur. Then for a given sample path we can find a $n_{0}$ such that none of these occur for $n \geqslant n_{0}$.

Let $[s, t]$ be an interval with $t_{N}-s_{N}=\min \left\{t_{p}-s_{p}\right\}$ and $\lambda([s, t]) \leqslant n_{0}^{N} 2^{-N n_{0}}$. There is clearly no loss of generality in the first assumption; the second implies that $t_{N}-s_{N} \leqslant n_{0} 2^{-n_{0}}$. Now we choose $n$ so that

$$
(n+1) 2^{-n-1}<t_{N}-s_{N} \leqslant n 2^{-n}
$$

and then $j, k, m$, and $i$ so that

$$
\begin{aligned}
& (j-1) 2^{-n} \leqslant s_{N}<j 2^{-n}, \quad(j+k) 2^{-n} \leqslant t_{N}<(j+k+1) 2^{-n}, \\
& 2^{-\left(m_{p}+1\right) / n}<t_{p}-s_{p} \leqslant 2^{-m_{p} / n}, \quad n^{-1} i_{p} 2^{-m_{p} / n} \leqslant s_{p}<n^{-1}\left(i_{p}+1\right) 2^{-m_{p} / n} .
\end{aligned}
$$

It is now easy to check that if $[s, t] \subset[0,1]^{N}$ the restrictions on the indices are satisfied and $[s, t]$ is one of the intervals in the event $E(i, j, k, m, n)$. This proves (3.1).

Next we show

$$
\liminf _{\varepsilon \rightarrow 0} I(\varepsilon) \geqslant 2^{1 / 2} \quad \text { a.s. }
$$

Note that it is sufficient to prove it for $d=1$ since $\|X([s, t])\|$ is larger than any of its component.

Let $\varepsilon_{n}=\theta^{-n N}, \theta>1, n \geqslant 1$. Note that for any $\varepsilon$ there is an integer $n$ such that $\varepsilon_{n+1}<\varepsilon \leqslant \varepsilon_{n}$. We have

$$
\begin{aligned}
\liminf _{\varepsilon \rightarrow 0} I(\varepsilon) & \geqslant \liminf _{n \rightarrow \infty} \inf _{\varepsilon_{n+1}<\varepsilon \leqslant \varepsilon_{n}} I(\varepsilon) \\
& \geqslant \liminf _{n \rightarrow \infty} \sup _{s, t \in[0,1]^{N}, \lambda([s, t]) \leqslant \varepsilon_{n+1}} \beta(s, t)^{-1}\left|X^{(N)}([s, t])\right| \\
& \geqslant \liminf _{n \rightarrow \infty} \max _{\langle 1\rangle \leqslant\left\langle i_{p}\right\rangle \leqslant\left\langle\theta^{n+1} / K\right\rangle} \beta\left(\langle 0\rangle,\left\langle\theta^{-(n+1)}\right\rangle\right)^{-1}\left|X^{(N)}\left(\left[\left\langle i_{p} K \theta^{-(n+1)}\right\rangle,\left\langle\left(i_{p} K+1\right) \theta^{-(n+1)}\right\rangle\right]\right)\right| \\
& =: J,
\end{aligned}
$$

where $K>0$ is a large constant which will be chosen later and $\left\langle f\left(i_{p}\right)\right\rangle$ means a $N$-dimensional vector $\left(f\left(i_{1}\right), \ldots\right.$, $\left.f\left(i_{N}\right)\right)$.

For any real numbers $t, H$, set $f(t)=(1+t)^{2 H}-t^{2 H}$. Then

$$
f^{\prime}(t)=2 H\left\{(1+t)^{2 H-1}-t^{2 H-1}\right\} .
$$

It follows that

$$
\left|f^{\prime}(t)\right| \leqslant 2|H(2 H-1)| t^{2 H-2} \vee(1+t)^{2 H-2} .
$$

Then, by fact (i), we have for $\left\langle i_{p}\right\rangle \neq\left\langle j_{p}\right\rangle$ 


$$
\begin{aligned}
E & {\left[X^{(N)}\left(\left[\left\langle i_{p} K \theta^{-(n+1)}\right\rangle,\left\langle\left(i_{p} K+1\right) \theta^{-(n+1)}\right\rangle\right]\right) X^{(N)}\left(\left[\left\langle j_{p} K \theta^{-(n+1)}\right\rangle,\left\langle\left(j_{p} K+1\right) \theta^{-(n+1)}\right\rangle\right]\right)\right] } \\
& =\frac{1}{2^{N}} \theta^{-2(n+1) \sum \alpha_{p}} \prod_{p=1}^{N}\left(-2\left(i_{p} K-j_{p} K\right)^{2 \alpha_{p}}+\left(i_{p} K-j_{p} K+1\right)^{2 \alpha_{p}}+\left(i_{p} K-j_{p} K-1\right)^{2 \alpha_{p}}\right) \\
& =\frac{1}{2^{N}} \theta^{-2(n+1) \sum \alpha_{p}} \prod_{p=1}^{N}\left(f\left(i_{p} K-j_{p} K\right)-f\left(i_{p} K-j_{p} K-1\right)\right) \\
& \leqslant \frac{1}{2^{N}} \theta^{-2(n+1) \sum \alpha_{p}} \prod_{p=1}^{N}\left|f^{\prime}\left(\xi_{p}\right)\right| \quad\left(\xi_{p} \in\left[i_{p} K-j_{p} K-1, i_{p} K-j_{p} K\right]\right) \\
& \leqslant c \theta^{-2(n+1) \sum \alpha_{p}} \prod_{p=1}^{N}\left(\left(i_{p} K-j_{p} K-1\right)^{2 \alpha_{p}-2} \vee\left(i_{p} K-j_{p} K+1\right)^{2 \alpha_{p}-2}\right) \\
& \leqslant c K^{\max \left(2 \alpha_{p}-2\right)} \theta^{-2(n+1) \sum \alpha_{p}}=: \rho(K) \delta_{n}^{2},
\end{aligned}
$$

where $\rho(K):=c K^{\max _{1 \leqslant p \leqslant N}\left(2 \alpha_{p}-2\right)}, \delta_{n}:=\theta^{-(n+1) \sum_{p=1}^{N} \alpha_{p}}$. Clearly, since $0<\alpha_{p}<1, \rho(K) \rightarrow 0$ as $K \rightarrow \infty$.

Let $\tau, \eta_{\left\langle i_{p}\right\rangle},\left\langle i_{p}\right\rangle \in \mathbb{Z}_{+}^{N}$, be independent mean zero Gaussian random variables with $E \tau^{2}=\rho(K) \delta_{n}^{2}, E \eta_{\left\langle i_{p}\right\rangle}^{2}=$ $(1-\rho(K)) \delta_{n}^{2}$. Let $\xi_{\left\langle i_{p}\right\rangle}=X^{(N)}\left(\left[\left\langle i_{p} K \theta^{-(n+1)}\right\rangle,\left\langle\left(i_{p} K+1\right) \theta^{-(n+1)}\right\rangle\right]\right)$. Then

$$
E\left[\left(\tau+\eta_{\left\langle i_{p}\right\rangle}\right)^{2}\right]=\delta_{n}^{2}=E\left[\xi_{\left\langle i_{p}\right\rangle}^{2}\right]
$$

and

$$
E\left[\xi_{\left\langle i_{p}\right\rangle} \xi_{\left\langle j_{p}\right\rangle}\right] \leqslant \rho(K) \delta_{n}^{2}=E\left[\left(\tau+\eta_{\left\langle i_{p}\right\rangle}\right)\left(\tau+\eta_{\left\langle j_{p}\right\rangle}\right)\right], \quad\left\langle i_{p}\right\rangle \neq\left\langle j_{p}\right\rangle .
$$

Thus, by Slepian lemma, for any $\mu>0$

$$
\begin{aligned}
& P\left(\max _{\langle 1\rangle \leqslant\left\langle i_{p}\right\rangle \leqslant\left\langle\theta^{n+1} / K\right\rangle} \xi_{\left\langle i_{p}\right\rangle} \leqslant(1-\mu) \delta_{n} \sqrt{2\left|\log \left(\varepsilon_{n+1}\right)\right|}\right) \\
& \quad \leqslant P\left(\max _{\langle 1\rangle \leqslant\left\langle i_{p}\right\rangle \leqslant\left\langle\theta^{n+1} / K\right\rangle}\left(\tau+\eta_{\left\langle i_{p}\right\rangle}\right) \leqslant(1-\mu) \delta_{n} \sqrt{2\left|\log \left(\varepsilon_{n+1}\right)\right|}\right) \\
& \quad \leqslant P\left(\max _{\langle 1\rangle \leqslant\left\langle i_{p}\right\rangle \leqslant\left\langle\theta^{n+1} / K\right\rangle} \eta_{\left\langle i_{p}\right\rangle} \leqslant\left(1-\frac{\mu}{2}\right) \delta_{n} \sqrt{2\left|\log \left(\varepsilon_{n+1}\right)\right|}\right)+P\left(-\tau \geqslant\left(\frac{\mu}{2}\right) \delta_{n} \sqrt{2\left|\log \left(\varepsilon_{n+1}\right)\right|}\right) \\
& \quad=\prod_{i_{1}=1}^{\theta^{n+1} / K} \theta_{i_{N}=1}^{\theta^{n+1} / K} P\left(\eta_{\left\langle i_{p}\right\rangle} \leqslant\left(1-\frac{\mu}{2}\right) \delta_{n} \sqrt{2\left|\log \left(\varepsilon_{n+1}\right)\right|}\right)+P\left(-\tau \geqslant\left(\frac{\mu}{2}\right) \delta_{n} \sqrt{2\left|\log \left(\varepsilon_{n+1}\right)\right|}\right) .
\end{aligned}
$$

Noting the following well known fact: for all $x>0$

$$
(2 \pi)^{-1 / 2}\left(1-x^{-2}\right) x^{-1} \mathrm{e}^{-x^{2} / 2} \leqslant P(N(0,1)>x) \leqslant(2 \pi)^{-1 / 2} x^{-1} \mathrm{e}^{-x^{2} / 2},
$$

where $N(0,1)$ is the standard normal random variable,

$$
\begin{aligned}
P\left(\eta_{\left\langle i_{p}\right\rangle} \leqslant\left(1-\frac{\mu}{2}\right) \delta_{n} \sqrt{2\left|\log \left(\varepsilon_{n+1}\right)\right|}\right) & =P\left(N(0,1) \leqslant\left(1-\frac{\mu}{2}\right) \frac{\sqrt{2\left|\log \left(\varepsilon_{n+1}\right)\right|}}{1-\rho(K)}\right) \\
& =1-P\left(N(0,1)>\left(1-\frac{\mu}{2}\right) \frac{\sqrt{2\left|\log \left(\varepsilon_{n+1}\right)\right|}}{1-\rho(K)}\right) \\
& \leqslant 1-\varepsilon_{n+1}^{(1-\mu / 4)^{2} /(1-\rho(K))^{2}} \leqslant \mathrm{e}^{-\varepsilon_{n+1}^{(1-\mu / 4)^{2} /(1-\rho(K))^{2}}}
\end{aligned}
$$

for large $n$, where for obtaining the last inequality the following basic fact has been used: $\forall x, 1-x \leqslant \mathrm{e}^{-x}$. Moreover

$$
P\left(-\tau \geqslant \frac{\mu}{2} \delta_{n} \sqrt{2\left|\log \left(\varepsilon_{n+1}\right)\right|}\right)=P\left(N(0,1) \geqslant \frac{\mu}{2} \frac{\sqrt{2\left|\log \left(\varepsilon_{n+1}\right)\right|}}{\rho(K)}\right) \leqslant c \varepsilon_{n+1}^{\mu^{2} /\left(4 \rho(K)^{2}\right)} .
$$


Thus we get that the right-hand side of (3.3) is bounded by

$$
\mathrm{e}^{-\theta^{(n+1) N} \varepsilon_{n+1}^{(1-\mu / 4)^{2} /(1-\rho(K))^{2}} / K^{N}}+c \varepsilon_{n+1}^{\mu^{2} /\left(4 \rho(K)^{2}\right)} .
$$

Since $\rho(K) \downarrow 0$, we can choose $K$ large enough such that $(1-\mu / 4)^{2} /(1-\rho(K))^{2}<1$. Thus the sum of the above bound is finite and hence the sum of the left-hand side of (3.3) is finite. By Borel-Cantelli lemma, we get

$$
\liminf _{n \rightarrow \infty} \max _{\langle 1\rangle \leqslant\left\langle i_{p}\right\rangle \leqslant\left\langle\theta^{n+1} / K\right\rangle} \frac{\xi_{\left\langle i_{p}\right\rangle}}{\delta_{n} \sqrt{\left|\log \left(\varepsilon_{n+1}\right)\right|}} \geqslant 2^{1 / 2} \text { a.s., }
$$

which (also noting $\beta\left(\langle 0\rangle,\left\langle\theta^{-(n+1)}\right\rangle\right)=\delta_{n} \sqrt{\left|\log \left(\varepsilon_{n+1}\right)\right|}$ ) implies $J \geqslant 2^{1 / 2}$ a.s. This proves (3.2). The proof of Theorem 3.1 is complete.

Theorem 3.2 (Global continuity modulus for points). Let

$$
h_{1}(\xi)=\xi^{\min \alpha_{i}}(2|\log \xi|)^{1 / 2}, \quad h_{2}(\xi)=\xi^{\max \alpha_{i}}(2|\log \xi|)^{1 / 2},
$$

where the minimum and the maximum are taken over all $1 \leqslant i \leqslant N$. Then

$$
\begin{aligned}
& \limsup _{\varepsilon \rightarrow 0} \sup _{s, t \in[0,1]^{N}, \delta(s, t) \leqslant \varepsilon} h_{1}(\delta(s, t))^{-1}\left\|X_{t}-X_{s}\right\| \leqslant N^{1 / 2} \quad \text { a.s., } \\
& \liminf _{\varepsilon \rightarrow 0} \sup _{s, t \in[0,1]^{N}, \delta(s, t) \leqslant \varepsilon} h_{2}(\delta(s, t))^{-1}\left\|X_{t}-X_{s}\right\| \geqslant N^{1 / 2} \text { a.s. }
\end{aligned}
$$

Remark 3.2. A special case is that if $\alpha_{1}=\cdots=\alpha_{N}=H$, (3.4) and (3.5) can be equalities. This special case is an extension of Theorem 2.4 of Orey and Pruitt [9] in fractional case.

Proof. The proof is obvious adaptation of the argument of Theorem 2.4 of Orey and Pruitt [9]. In order to show (3.4), in the proof of upper bound of Theorem 2.4 of Orey and Pruitt [9], it suffices to replace the use of Theorem 2.1 of Orey and Pruitt [9] by that of our Theorem 3.1, and replace the function $h(\cdot)$ by $h_{1}(\cdot)$.

In order to show (3.5), in the proof of lower bound of Theorem 2.4 of Orey and Pruitt [9], it suffices to replace $A_{m k}, B_{m j}$ by the following $A_{m k}^{\prime}, B_{m j}^{\prime}$ respectively:

$$
\begin{aligned}
& A_{m k}^{\prime}=\left[\left(\frac{1}{2}+\frac{k K}{m} \eta, 0\right),\left(\frac{1}{2}+\frac{k K+1}{m} \eta, \frac{1}{2}\right)\right], \quad k=0, \ldots, m-1 ; m=1,2, \ldots, \\
& B_{m j}^{\prime}=\left[\left(0, \frac{1}{2}+\frac{j K}{m} \eta\right),\left(\frac{1}{2}, \frac{1}{2}+\frac{j K+1}{m} \eta\right)\right], \quad j=0, \ldots, m-1 ; m=1,2, \ldots
\end{aligned}
$$

(where $K$ is a large constant) and replace the function $h(\cdot)$ by $h_{2}(\cdot)$ because, following the second part proof of Theorem 3.1 (see the proof of (3.2)), (2.14) and (2.15) of Orey and Pruitt [9] hold with $h(\cdot)$ replaced by $h_{2}(\cdot)$ and $A_{m k}, B_{m j}$ replaced by $A_{m k}^{\prime}, B_{m j}^{\prime}$ respectively. This completes the proof.

\section{Local moduli of continuity, laws of the iterated logarithm}

In this section we shall show the local continuity moduli for $X:=X^{(N, d)}$.

Theorem 4.1 (Local continuity modulus for intervals; LIL). Let $s \in[0,1]^{N}$. Then

$$
\limsup _{\varepsilon \rightarrow 0} \sup _{t \in[0,1]^{N}, \lambda([s, t]) \leqslant \varepsilon} \gamma(s, t)^{-1}\|X([s, t])\|=(2 N)^{1 / 2} \quad \text { a.s., }
$$

where

$$
\gamma(s, t)=d(s, t)\left(\log \log \left(\lambda([s, t])^{-1}\right)\right)^{1 / 2},
$$

here $\lambda([s, t]), d(s, t)$ are defined as above. Particularly,

$$
\limsup _{\varepsilon \rightarrow 0} \sup _{t \in[0,1]^{N}, \lambda([\langle 0\rangle, t]) \leqslant \varepsilon} \gamma(\langle 0\rangle, t)^{-1}\left\|X_{t}\right\|=(2 N)^{1 / 2} \quad \text { a.s. }
$$


Remark 4.1. When $N=2, d=1, \alpha_{1}=\alpha_{2}=1 / 2$, (4.2) is the well-known law of the iterated logarithm (LIL) of 2-parameter Wiener processes (cf. Theorem 1.12.2 in Csörgó and Révész [3]).

Proof. By fact (iii) (4.2) is equivalent to (4.1). So for convenience sake it is enough to show (4.2). We first show

$$
\text { the left-hand side of }(4.2) \leqslant(2 N)^{1 / 2} \text { a.s. }
$$

The proof can be given in a parallel way to the first part proof of Theorem 3.1. Let $m=\left(m_{1}, \ldots, m_{N-1}\right)$, and define the events for $\mu>0$

$$
F(k, m, n)=\left[\sup \left\|X_{t}\right\| \geqslant(1+\mu) A \sqrt{2 N \log \log \left(B^{-1}\right)}\right],
$$

where the supremum is taken over all $t$ satisfying

$$
\begin{aligned}
& 2^{-\left(m_{p}+1\right) / \log n} \leqslant t_{p}<2^{-m_{p} / \log n}, \quad p=1, \ldots, N-1, \\
& k 2^{-n} \leqslant t_{N}<(k+1) 2^{-n},
\end{aligned}
$$

and

$$
A=A(\alpha, k, m, n)=2^{-\left(\alpha_{1}\left(m_{1}+1\right)+\cdots+\alpha_{N-1}\left(m_{N-1}+1\right)\right) / \log n}\left(k 2^{-n}\right)^{\alpha_{N}}
$$

is the infimum of $d(\langle 0\rangle, t)$ for the intervals involved, and

$$
A^{\prime}=A^{\prime}(\alpha, k, m, n)=2^{-\left(\alpha_{1} m_{1}+\cdots+\alpha_{N-1} m_{N-1}\right) / \log n}\left((k+1) 2^{-n}\right)^{\alpha_{N}}
$$

is the corresponding supremum, and

$$
B=B(\alpha, k, m, n)=2^{-\left(m_{1}+\cdots+m_{N-1}\right) / \log n}(k+1) 2^{-n}
$$

is the supremum of $\lambda([\langle 0\rangle, t])$ for the intervals involved. The parameters are restricted to the following ranges:

$$
\frac{1}{4} \log n \leqslant k \leqslant \log n, \quad 0 \leqslant m_{p}<n \log n, \quad n=3,4, \ldots
$$

It is easy to check that

$$
0 \leqslant 1-A\left(A^{\prime}\right)^{-1} \leqslant c(\log n)^{-1} \rightarrow 0 .
$$

By (2.3) (with $\left.u=(1+\mu) A\left(A^{\prime}\right)^{-1} \sqrt{2 N \log \log \left(B^{-1}\right)}\right), \delta(t)=\prod_{i} t_{i}$ and $B \leqslant(\log n+1) 2^{-n}$, we obtain

$$
\begin{aligned}
P(F(k, m, n)) & \leqslant c \exp \left[-\left(1+\frac{\mu}{2}\right)^{2} N \log \log \left(B^{-1}\right)\right] \\
& \leqslant c n^{-(1+\mu / 2)^{2} N}
\end{aligned}
$$

for large $n$. Thus

$$
\sum_{n} \sum_{k, m} P(F(k, m, n)) \leqslant c \sum_{n=1}^{\infty}(\log n)^{N} n^{-(1+\mu / 2)^{2} N+N-1}<\infty .
$$

Thus, by Borel-Cantelli lemma, a.s. only finitely many of the $F(k, m, n)$ occur. Then for a given sample path we can find a $n_{0}$ such that none of these occur for $n \geqslant n_{0}$.

Let $t \in[0,1]^{N}$ be a point with $t_{N}=\min \left\{t_{p}\right\}$ and $\delta(t) \leqslant\left(\log n_{0}\right)^{N} 2^{-N n_{0}}$. There is clearly no loss of generality in the first assumption; the second implies that $t_{N} \leqslant\left(\log n_{0}\right) 2^{-n_{0}}$. Now we choose $n$ so that

$$
(\log (n+1)) 2^{-n-1}<t_{N} \leqslant(\log n) 2^{-n}
$$

and then $k, m$, so that

$$
k 2^{-n} \leqslant t_{N}<(k+1) 2^{-n}, \quad 2^{-\left(m_{p}+1\right) / \log n}<t_{p} \leqslant 2^{-m_{p} / \log n} .
$$

It is now easy to check that if $t \in[0,1]^{N}$ the restrictions on the indices are satisfied and $t$ is one of the point in the event $F(k, m, n)$. This proves (4.3). 
Now we show the opposite inequality of (4.3). Note that it is enough to show it for $d=1$ since $\left\|X_{t}\right\|$ is larger than any of its component. Let $m=\left(m_{1}, \ldots, m_{N-1}\right)$ where components restrictions

$$
n \log n \leqslant m_{p}<n \log n+n, \quad p=1, \ldots, N-1, n=3,4, \ldots,
$$

so that all values of $m_{1}+\cdots+m_{N-1}$ are different. Clearly, the combinatorial number of possible vectors $\left(m_{1}, \ldots, m_{N-1}\right)$ is at least

$$
C_{N-1}^{n}=\frac{n !}{(N-1) !(n-N+1) !} \sim \frac{n^{N-1}}{(N-1) !} \quad \text { as } n \rightarrow \infty .
$$

Fix such $m$. Let

$$
t_{1}=\cdots=t_{N-1}=2^{-\left(\left(m_{1}+\cdots+m_{N-1}\right) /(N-1) \log n\right)^{q}}, \quad t_{N}=2^{-n^{q}},
$$

where $q>1$ is a constant which will be specified later. For such $t$, let

$$
\gamma(t)=\gamma(\langle 0\rangle, t)=d(\langle 0\rangle, t)\left(\log \log \left(\delta(t)^{-1}\right)\right)^{1 / 2} .
$$

It is sufficient to show

$$
\limsup _{n} \max _{m} \gamma(t)^{-1}\left|X_{t}^{(N)}\right| \geqslant(2 N)^{1 / 2} \quad \text { a.s. }
$$

Define the events for $0<\mu<1$

$$
G(m, n, \mu)=\left[\gamma(t)^{-1}\left|X_{t}^{(N)}\right| \geqslant(1-\mu)(2 N)^{1 / 2}\right] .
$$

By (2.4) and $\delta(t)=\prod_{i} t_{i} \geqslant 2^{-2(2 n)^{q}}$ we get

$$
\begin{aligned}
P(G(m, n, \mu)) & =P\left[|N(0,1)| \geqslant(1-\mu) \sqrt{2 N \log \log \left(\delta(t)^{-1}\right)}\right] \\
& \geqslant c\left(\log \log \left(\delta(t)^{-1}\right)\right)^{-1} \exp \left[-(1-\mu)^{2} N \log \log \left(\delta(t)^{-1}\right)\right] \\
& \geqslant c n^{-(1-\mu / 2)^{2} q N}
\end{aligned}
$$

for large $n$, where $N(0,1)$ is a standard normal random variable. Thus

$$
\begin{aligned}
\sum_{n} \sum_{m} P(G(m, n, \mu)) & \geqslant c \sum_{n=1}^{\infty} \sum_{m} n^{-(1-\mu / 2)^{2} q N} \geqslant c \sum_{n=1}^{\infty} C_{N-1}^{n} n^{-(1-\mu / 2)^{2} q N} \\
& \geqslant c \sum_{n=1}^{\infty} n^{-(1-\mu / 2)^{2} q N+N-1}=\infty
\end{aligned}
$$

by choosing $q>1$ such that $-(1-\mu / 2)^{2} q+1>0$, where $\sum_{m}$ takes over the above ranges of $m$.

In order to get the desired result, we need to solve dependence problems. To do this, we will use (2.6) the stochastic integral representation of the process $X^{(N)}$.

Let $a_{n}=n 2^{n^{q}}, n \geqslant 1$. To solve dependence-relation problems, we consider the process

$$
\bar{X}_{n}^{(N)}(t):=\bar{X}^{(N)}\left(t, a_{n-1}, a_{n}\right), \quad \widetilde{X}_{n}^{(N)}(t):=X_{t}^{(N)}-\bar{X}^{(N)}\left(t, a_{n-1}, a_{n}\right),
$$

where

$$
\bar{X}^{(N)}\left(t, a_{n-1}, a_{n}\right)=\int_{\left|x_{N}\right| \in\left(a_{n-1}, a_{n}\right)} \int_{\mathbb{R}^{N-1}} \prod_{i=1}^{N} f\left(\alpha_{i}, t_{i}, x_{i}\right) \mathrm{d} W^{(N)}(x) .
$$

Here, and in the sequel of this section, $f$ is defined as in (2.7). Define events for $0<\mu<1$

$$
\begin{aligned}
& A_{n}(\mu)=\left[\max _{m} \gamma(t)^{-1}\left|X_{t}^{(N)}\right| \geqslant(1-\mu)(2 N)^{1 / 2}\right], \\
& B_{n}(\mu)=\left[\max _{m} \gamma(t)^{-1}\left|\bar{X}_{n}^{(N)}(t)\right| \geqslant(1-\mu)(2 N)^{1 / 2}\right], \\
& C_{n}(\mu)=\left[\max _{m} \gamma(t)^{-1}\left|\widetilde{X}_{n}^{(N)}(t)\right| \geqslant \mu(2 N)^{1 / 2}\right],
\end{aligned}
$$


where $t, m$ are defined as above. Clearly,

$$
A_{n}(\mu) \subset B_{n}(2 \mu) \cup C_{n}(\mu) \subset A_{n}(3 \mu) \cup C_{n}(\mu),
$$

and $\bar{X}_{n}^{(N)}(\cdot), n=3,4, \ldots$, are independent due to the virtue of independent increments of $N$-parameter Wiener processes and hence $\left\{B_{n}(\mu)\right\}$ are independent. By standard Borel-Cantelli lemma argument, in order to get (4.4) it is enough to show

$$
\sum_{n=1}^{\infty} P\left(B_{n}(\mu)\right)=\infty
$$

and

$$
\sum_{n=1}^{\infty} P\left(C_{n}(\mu)\right)<\infty .
$$

For any $t$, it holds (by isometry property, see Lemma 3 of Park [11])

$$
\begin{aligned}
E\left[\widetilde{X}_{n}^{(N)}(t)^{2}\right] & =\int_{\left|x_{N}\right| \notin\left(a_{n-1}, a_{n}\right)} \int_{\mathbb{R}^{N-1}} \prod_{i=1}^{N} f\left(\alpha_{i}, t_{i}, x_{i}\right)^{2} \mathrm{~d} x_{1} \cdots \mathrm{d} x_{N} \\
& =\prod_{i=1}^{N-1} t_{i}^{2 \alpha_{i}} \int_{\left|x_{N}\right| \notin\left(a_{n-1}, a_{n}\right)} f\left(\alpha_{N}, t_{N}, x_{N}\right)^{2} \mathrm{~d} x_{N} \\
& \leqslant 4 c\left(\alpha_{N}\right)^{2} \prod_{i=1}^{N-1} t_{i}^{2 \alpha_{i}}\left(\int_{\left|x_{N}\right| \leqslant a_{n-1}}+\int_{\left|x_{N}\right| \geqslant a_{n}}\right)\left(1-\cos \left(t_{N} x_{N}\right)\right) \frac{\mathrm{d} x_{N}}{\left|x_{N}\right|^{2 \alpha_{N}+1}} \\
& \leqslant c \prod_{i=1}^{N-1} t_{i}^{2 \alpha_{i}}\left(t_{N}^{2} a_{n-1}^{2-2 \alpha_{N}}+a_{n}^{-2 \alpha_{N}}\right) \\
& \leqslant c d(t)\left(\left(t_{N} a_{n-1}\right)^{2-2 \alpha_{N}}+\left(t_{N} a_{n}\right)^{-2 \alpha_{N}}\right) .
\end{aligned}
$$

To get the second inequality from bottom, in the first integral we bound $1-\cos (t x)$ by $|t|^{2}|x|^{2}$, and the second one by 2 to get the required bound. Thus, since $0<\alpha_{N}<1$,

$$
t_{N} a_{n-1} \leqslant(n-1) 2^{-q(n-1)^{q-1}} \leqslant n^{-2 \alpha_{N} /\left(2-2 \alpha_{N}\right)} \quad \text { for large } n, \quad t_{N} a_{n}=n,
$$

we have

$$
E\left[\tilde{X}_{n}^{(N)}(t)^{2}\right] \leqslant c d(t) n^{-2 \alpha_{N}}
$$

for large $n$. It follows easily that

$$
\sum_{n} P\left(C_{n}(\mu)\right) \leqslant \sum_{n} \sum_{m} P\left(\gamma(t)^{-1}\left|\tilde{X}_{n}^{(N)}(t)\right| \geqslant \mu(2 N)^{1 / 2}\right)<\infty .
$$

This proves (4.7).

We now show (4.6). Let $b_{1}(m)=g\left(m_{1}+\cdots+m_{N-1}-1\right), b_{2}(m)=g\left(m_{1}+\cdots+m_{N-1}\right)$, where $g(x)=$ $x 2^{(x /((N-1) \log n))^{q}}$. To get further independence relation, we consider the process $\bar{X}_{n}^{(N)}(t, m):=\bar{X}^{(N)}\left(t, a_{n-1}, a_{n}, m\right)$, $\widetilde{X}_{n}^{(N)}(t, m):=\bar{X}_{n}^{(N)}(t)-\bar{X}^{(N)}\left(t, a_{n-1}, a_{n}, m\right)$ where

$$
\begin{aligned}
& \bar{X}^{(N)}\left(t, a_{n-1}, a_{n}, m\right) \\
& =\int_{\left|x_{1}\right| \in\left(b_{1}(m), b_{2}(m)\right)} \int_{\left|x_{N}\right| \in\left(a_{n-1}, a_{n}\right)} \int_{\mathbb{R}^{N-2}} \prod_{i=1}^{N} f\left(\alpha_{i}, t_{i}, x_{i}\right) \mathrm{d} W^{(N)}(x) .
\end{aligned}
$$


Fixed $n$. Clearly, $\bar{X}_{n}^{(N)}(t, m)$ are independent for such $m$ so that all values of $m_{1}+\cdots+m_{N-1}$ are different. Define events for $0<\mu<1$

$$
\begin{aligned}
& B_{n}^{\prime}(\mu)=\left[\max _{m} \gamma(t)^{-1}\left|\bar{X}_{n}^{(N)}(t, m)\right| \geqslant(1-\mu)(2 N)^{1 / 2}\right], \\
& B_{n}^{\prime \prime}(\mu)=\left[\max _{m} \gamma(t)^{-1}\left|\tilde{X}_{n}^{(N)}(t, m)\right| \geqslant \mu(2 N)^{1 / 2}\right] .
\end{aligned}
$$

Clearly,

$$
B_{n}(\mu) \subset B_{n}^{\prime}(2 \mu) \cup B_{n}^{\prime \prime}(\mu) \subset B_{n}(3 \mu) \cup B_{n}^{\prime \prime}(\mu) .
$$

Since $n \leqslant\left(m_{1}+\cdots+m_{N-1}\right) /(N-1) \log n \leqslant n+n / \log n$, we have

$$
\begin{aligned}
t_{1} b_{1}(m) & \leqslant\left(m_{1}+\cdots+m_{N-1}-1\right) 2^{-q\left(\left(m_{1}+\cdots+m_{N-1}-1\right) /(N-1) \log n\right)^{q-1}((N-1) \log n)^{-1}} \\
& \leqslant c n 2^{-c n^{q-1}} \leqslant n^{-2 \alpha_{1} /\left(2-2 \alpha_{1}\right)} \quad(\text { for large } n), \\
t_{1} b_{2}(m) & =\left(m_{1}+\cdots+m_{N-1}\right) \geqslant(N-1) n \log n,
\end{aligned}
$$

where for obtaining the first inequality we have used the mean value theorem. Thus, similarly to (4.8) we have

$$
E\left[\widetilde{X}_{n}^{(N)}(t, m)^{2}\right] \leqslant c d(t) n^{-2 \alpha_{1}}
$$

for large $n$ and any $m$ defined above. It follows easily that

$$
\sum_{n} P\left(B_{n}^{\prime \prime}(\mu)\right) \leqslant \sum_{n} \sum_{m} P\left(\gamma(t)^{-1}\left|\tilde{X}_{n}^{(N)}(t, m)\right| \geqslant \mu(2 N)^{1 / 2}\right)<\infty .
$$

Noting

$$
\bar{X}_{n}^{(N)}(t, m)=\bar{X}_{n}^{(N)}(t)-\widetilde{X}_{n}^{(N)}(t, m)=X_{t}^{(N)}-\widetilde{X}_{n}^{(N)}(t)-\widetilde{X}_{n}^{(N)}(t, m),
$$

we have

$$
\begin{aligned}
\sum_{m} P\left(\gamma(t)^{-1}\left|\bar{X}_{n}^{(N)}(t, m)\right| \geqslant(1-\mu)(2 N)^{1 / 2}\right) \geqslant & \sum_{m} P\left(\gamma(t)^{-1}\left|X_{t}^{(N)}\right| \geqslant\left(1-\frac{\mu}{2}\right)(2 N)^{1 / 2}\right) \\
& -\sum_{m} P\left(\gamma(t)^{-1}\left|\tilde{X}_{n}^{(N)}(t)\right| \geqslant \frac{\mu}{4}(2 N)^{1 / 2}\right) \\
& -\sum_{m} P\left(\gamma(t)^{-1}\left|\tilde{X}_{n}^{(N)}(t, m)\right| \geqslant \frac{\mu}{4}(2 N)^{1 / 2}\right) \\
\geqslant & c n^{-(1-\mu / 8)^{2} q N+N-1}
\end{aligned}
$$

by (4.5), (4.7) (see its proof) and (4.9). Thus, since, for fixed $n, \bar{X}_{n}^{(N)}(t, m)$ are independent for $m$ such that $m_{1}+\cdots+m_{N-1}$ are different, we have

$$
\begin{aligned}
P\left(B_{n}^{\prime}(\mu)\right) & =1-P\left(\max _{m} \gamma(t)^{-1}\left|\bar{X}_{n}^{(N)}(t, m)\right|<(1-\mu)(2 N)^{1 / 2}\right) \\
& =1-\prod_{m}\left[1-P\left(\gamma(t)^{-1}\left|\bar{X}_{n}^{(N)}(t, m)\right| \geqslant(1-\mu)(2 N)^{1 / 2}\right)\right] \\
& \geqslant 1-\exp \left[-\sum_{m} P\left(\gamma(t)^{-1}\left|\bar{X}_{n}^{(N)}(t, m)\right| \geqslant(1-\mu)(2 N)^{1 / 2}\right)\right] \\
& \geqslant 1-\exp \left[-c n^{-(1-\mu / 8)^{2} q N+N-1}\right] .
\end{aligned}
$$

Choosing $q>1$ small enough again such that $-(1-\mu / 8)^{2} q+1>0$, and using the following relation: $1-\mathrm{e}^{-x} \geqslant x / 2$ for $0 \leqslant x \leqslant \log 2$, we get that the last term $\geqslant c n^{-(1-\mu / 8)^{2} q N+N-1}$ for $n$ large enough. Thus, $\sum_{n} P\left(B_{n}^{\prime}(\mu)\right)$ is infinite. This proves (4.6). The proof of Theorem 4.1 is now complete.

The following form of LIL is parallel to that of a standard fractional Brownian motion (cf. Ortega [10] or Monrad and Rootzén [8]). Its proof is easier than that of the last theorem and is standard. So we omit the proof details here. 
Theorem 4.2 (LIL). Let $\zeta(\varepsilon)=\varepsilon^{\sum_{i=1}^{N} \alpha_{i}}\left(\log \log \left(\varepsilon^{-1}\right)\right)^{1 / 2}$. Then

$$
\limsup _{\varepsilon \rightarrow 0} \sup _{\langle 0\rangle \leqslant\left\langle t_{i}\right\rangle \leqslant\langle\varepsilon\rangle} \zeta(\varepsilon)^{-1}\left\|X_{t}\right\|=\limsup _{\varepsilon \rightarrow 0} \zeta(\varepsilon)^{-1}\left\|X_{\langle\varepsilon\rangle}\right\|=2^{1 / 2} \quad \text { a.s. }
$$

\section{Acknowledgement}

The author wishes to express his deep gratitude to an anonymous referee for his valuable comments on an earlier version which improve the quality of this paper.

\section{Appendix A}

Proofs of Facts (i)-(v). We first show (2.1). By definition (see Section 1), the $X^{(N)}$-measure of interval $[s, t]$ of $\mathbb{R}_{+}^{N}$

$$
X^{(N)}([s, t])=\Delta_{t_{N}, s_{N}}^{(N)} \Delta_{t_{N-1}, s_{N-1}}^{(N-1)} \cdots \Delta_{t_{1}, s_{1}}^{(1)} X^{(N)},
$$

where

$$
\Delta_{t_{i}, s_{i}}^{(i)} X^{(N)}(x)=X\left(x_{1}, \ldots, x_{i-1}, t_{i}, x_{i+1}, \ldots, x_{N}\right)-X\left(x_{1}, \ldots, x_{i-1}, s_{i}, x_{i+1}, \ldots, x_{N}\right) .
$$

To simplify notation, let $\Gamma(s, t, y)=\Delta_{t_{N-1}, s_{N-1}}^{(N-1)} \cdots \Delta_{t_{1}, s_{1}}^{(1)} X^{(N)}\left(x_{1}, \ldots, x_{N-1}, y\right), s, t \in \mathbb{R}^{N-1}, y \in \mathbb{R}^{1}$. Then for $s, t \in \mathbb{R}^{N-1}$

$$
X^{(N)}\left([s, t] \times\left[s_{N}, t_{N}\right]\right)=\Gamma\left(s, t, t_{N}\right)-\Gamma\left(s, t, s_{N}\right) .
$$

Note that (by (1.1))

$$
E\left[\Gamma\left(s, t, t_{N}\right) \Gamma\left(s^{\prime}, t^{\prime}, t_{N}^{\prime}\right)\right]=2^{-1}\left(\left|t_{N}\right|^{2 \alpha_{N}}+\left|t_{N}^{\prime}\right|^{2 \alpha_{N}}-\left|t_{N}-t_{N}^{\prime}\right|^{2 \alpha_{N}}\right) E\left[X^{(N-1)}([s, t]) X^{(N-1)}\left(\left[s^{\prime}, t^{\prime}\right]\right)\right] .
$$

Thus, by induction, (2.1) is verified immediately.

Facts (ii)-(v) are immediate consequences of (2.1).

\section{References}

[1] E. Belinsky, W. Linde, Small ball probabilities of fractional Brownian sheets via fractional integration operators, J. Theor. Probab. 15 (2002) 589-612.

[2] E. Csáki, M. Csörgő, Q.-M. Shao, Fernique type inequalities and moduli of continuity for $l^{2}$-valued Ornstein-Uhlenbeck processes, Ann. Inst. H. Poincaré Probab. Statist. 28 (1992) 479-517.

[3] M. Csörgô, P. Révész, Strong Approximations in Probability and Statistics, Academic Press, New York, 1981.

[4] T. Dunker, Estimates for the small probabilities of the fractional Brownian sheet, J. Theor. Probab. 13 (2000) 357-382.

[5] W. Ehm, Sample function properties of multi-parameter stable processes, Z. Wahrsch. Verw. Gebiete 96 (1981) $195-228$.

[6] C. Lu, Y. Wang, L.-X. Zhang, On large increments of a two-parameter fractional Wiener process, Sci. China 51 (2001) $215-223$.

[7] D.M. Mason, Z. Shi, Small deviations for some multi-parameter Gaussian processes, J. Theor. Probab. 14 (2001) $213-239$.

[8] D. Monrad, H. Rootzén, Small values of Gaussian processes and functional laws of the iterated logarithm, Probab. Theory Rel. Fields 101 (1995) 173-192.

[9] S. Orey, W.E. Pruitt, Sample functions of the $N$-parameter Wiener process, Ann. Probab. 1 (1973) 138-163.

[10] J. Ortega, On the size of the increments of non-stationary Gaussian processes, Stoch. Process. Appl. 18 (1984) 47-56.

[11] W.J. Park, A multi-parameter Gaussian process, Ann. Math. Statist. 41 (1970) 1582-1595.

[12] R. Pyke, Partial sums of matrix arrays and Brownian sheet, in: E.F. Harding, D.G. Kendall (Eds.), Stochastic Analysis, Wiley, New York, 1972, pp. 331-348.

[13] M. Talagrand, Multiple points of trajectories of multiparameter fractional Brownian motion, Probab. Theory Rel. Fields 112 (1998) $545-563$. 